\title{
Ophthalmology examinations under anaesthesia carried out on non-ophthalmology lists in a specialist children's hospital
}

\author{
Ivan Yeu Ming Yip $\mathbb{1}^{1} \cdot$ Filofteia Tacea ${ }^{1} \cdot$ Rutika Dodeja $^{1} \cdot$ Jodie Pendlebury ${ }^{1} \cdot$ Damien C. M. Yeo $\mathbb{1}^{1}$
}

Received: 28 December 2020 / Revised: 15 January 2021 / Accepted: 16 February 2021 / Published online: 5 March 2021

(c) The Author(s), under exclusive licence to The Royal College of Ophthalmologists 2021

\section{To the Editor:}

Recent advances in technology such as optical coherence tomography, wideview imaging and the iCare tonometer have made more accurate diagnosis in children possible [1]. However, there are times where an examination under anaesthesia (EUA) is still necessary. These children often have behavioural issues due to underlying comorbidities requiring multiple specialist input at dedicated paediatric hospitals.

To reduce the risks of repeated general anaesthetic [2] ophthalmologists carry out their examinations while a child is anaesthetised for another reason; i.e. neuroimaging. This is unique and an under-recognised aspect of paediatric ophthalmology. We present data of ophthalmic examinations taking place in children undergoing a general anaesthetic for non-ophthalmic issues.

A retrospective case note review of all children undergoing an ophthalmic EUA in a single paediatric hospital in the United Kingdom (UK) on any non-ophthalmic list between January 2017 to July 2019 inclusive was performed. Information collected included age, underlying diagnosis, location, grade of ophthalmologist and procedures undertaken. No children were excluded.

Sixty-five children underwent an EUA on a nonophthalmic list. Mean age was 5.4 years old $(\mathrm{SD} \pm 5.08$ years) (range 14 weeks-23 years old). The most common diagnosis was tuberous sclerosis $(21.5 \%, n=13)$. The full list of diagnoses can be found in Table 1 .

Fundal examination was carried out in 53 children $(81.5 \%)$ and anterior segment examination in 43 of them (66\%). Cycloplegic retinoscopy was required in 23 children

Ivan Yeu Ming Yip

Ivan.yip@nhs.net

1 Alder Hey Children's Hospital Ophthalmology Department, Liverpool, UK
(35.3\%) and $12(18.5 \%)$ had an IOP measurement. During 7 $(11 \%)$ of the EUAs, an ophthalmology procedure was carried out in the same sitting and this included 4 syringe and probing, 1 meibomian gland expression, 1 botulinum toxin for a squint and 1 cleaning an artificial eye.

Forty-two children (64.6\%) had an examination during a magnetic resonance imaging (MRI) scan. Twenty-three $(35 \%)$ examinations took place in theatre. The reasons for general anaesthetic were varied and ranged across 14 different specialities.

Twenty-seven (42\%), 22 (34.4\%) and 14 (20.3\%) examinations were performed by fellows, trainees and consultants, respectively. This is the first article reporting ophthalmic EUAs on non-ophthalmic lists. Approximately four EUAs take place every 5 weeks in a tertiary paediatric hospital.

Table 1 Reasons for eye examination under anaesthesia.

\begin{tabular}{lc}
\hline Reason for eye examination & Number $n=65(\%)$ \\
\hline Tuberous sclerosis complex & $13(20 \%)$ \\
Brain tumour/Chiari malformation/headaches & $7(10.7 \%)$ \\
Neurofibromatosis Type 1 & $7(10.7 \%)$ \\
Strabismus & $6(9.2 \%)$ \\
Systemic Syndrome ${ }^{a}$ & $5(7.7 \%)$ \\
Autistic Spectrum Disorder & $4(6.2 \%)$ \\
Haemangioma/Sturge Weber & $4(6.2 \%)$ \\
Epiphora & $4(6.2 \%)$ \\
Eye tumour & $3(4.6 \%)$ \\
Microphthalmia & $3(4.6 \%)$ \\
Developmental delay & $2(3.1 \%)$ \\
Skull abnormality & $2(3.1 \%)$ \\
Stickler Syndrome & $2(3.1 \%)$ \\
Meibomian Gland Disease & $1(1.5 \%)$ \\
Retinopathy of Prematurity & $1(1.5 \%)$ \\
Trauma & $1(1.5 \%)$
\end{tabular}

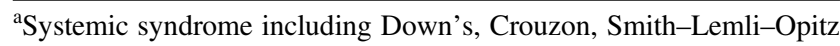
and unknown. 
These examinations are an under-recognised area of service provision. For these examinations to occur, significant administrative work is required. They are time critical having to be done in conjunction with the child's other appointment. For the examining doctor, they are labour and time intensive as they need to leave a clinic immediately, travel to the area with the equipment, consent and perform the examination. The doctor may attend too early only to have to return shortly after.

The EUA is the only way to gain a high-quality examination in a challenging child. They reduce the overall number and stress of clinic examinations. The COVID-19 pandemic has meant all EUAs occur with full personalprotective-equipment and this is safer than a difficult closeup examination in a distressed child.

Paediatric ophthalmologists in the UK are in short supply [3]. Other health professionals such as specialist nurses have taken on roles such as retinopathy of prematurity screening [4]. To assist in the workload, one can envisage a trained optometrist carrying out an EUA with consultant support.

Author contributions I.Y.M.Y. was responsible for analysing data, interpreting results and writing up the article. F.T. contributed by extracting and writing data. R.D. was involved in extracting data. J.P. contributed by keeping examination databases up-to-date. D.Y. was responsible for the idea of the study, guiding the other authors through data collection and interpreting results. In addition, he provided feedback and editing of the report.

\section{Compliance with ethical standards}

Conflict of interest The authors declare no competing interests.

Publisher's note Springer Nature remains neutral with regard to jurisdictional claims in published maps and institutional affiliations.

\section{References}

1. Biswas S, Gray J, Stanga P. Optos ${ }^{\circledR}$ widefield retinal imaging in a paediatric vitreoretinal service. Invest Ophthalmol Vis Sci 2012;53:1164.

2. Bartels DD, McCann ME, Davidson AJ, Polaner DM, Whitlock EL, Bateman BT. Estimating pediatric general anesthesia exposure: quantifying duration and risk. Paediatr Anaesth 2018;28: 520-527. https://doi.org/10.1111/pan.13391.

3. The Royal College of Ophthalmologists. New RCOphth Workforce Census illustrates the severe shortage of eye doctors in the UK. https://www.rcophth.ac.uk/2019/01/new-rcophth-workforcecensus-illustrates-the-severe-shortage-of-eye-doctors-in-the-uk/. Accessed 30 Sep 2020.

4. Dai Shuan, Shah Shaheen, Wu Zhen, Iverson Sharron. Specialist nurse screening for retinopathy of prematurity - a pilot study. AsiaPac J Ophthalmol. 2013;2:300-304. 